\title{
Comparison between an exclusive in vitro-produced embryo transfer system and artificial insemination for genetic, technical, and financial herd performance
}

\author{
K. Kaniyamattam, J. Block, P. J. Hansen, and A. De Vries ${ }^{1}$ \\ Department of Animal Sciences, University of Florida, Gainesville 32611
}

\begin{abstract}
The objective of this study was to implement an in vitro-produced embryo transfer (IVP-ET) system in an existing stochastic dynamic dairy simulation model with multitrait genetics to evaluate the genetic, technical, and financial performance of a dairy herd implementing an exclusive IVP-ET or artificial insemination (AI) system. In the AI system, sexed semen was used on the genetically best heifers only. In the IVP-ET system, all of the animals in the herd were impregnated with female sexed embryos created through in vitro fertilization of oocytes collected from animals of superior genetics for different traits of interest. Each donor was assumed to yield on average 4.25 transferable embryos per collection. The remaining animals in the herd were used as recipients and received either a fresh embryo or a frozen embryo when fresh embryos were not available. Selection of donors was random or based on the greatest estimated breeding value (EBV) of lifetime net merit (NM\$), milk yield, or daughter pregnancy rate. For both the IVP-ET and AI systems, culling of surplus heifer calves not needed to replace culled cows was based on the lowest EBV for the same traits. A herd of 1,000 milking cows was simulated 15 yr over time after the start of the IVP-ET system. The default cost to produce and transfer 1 embryo was set at $\$ 165$. Prices of fresh embryos at which an exclusive IVP-ET system financially breaks even with the comparable AI system in yr 15 and for an investment period of 15 yr were also estimated. More surplus heifer calves were sold from the IVP-ET systems than from the comparable AI systems. The surplus calves from the IVP-ET systems were also genetically superior to the surplus calves from the comparable AI systems, which might be reflected in their market value as a premium price. The most profitable scenario among the 4 IVP-ET scenarios in
\end{abstract}

Received September 8, 2016.

Accepted February 12, 2017.

${ }^{1}$ Corresponding author: devries@ufl.edu yr 15 was the one in which $\mathrm{NM} \$$ was maximized in the herd. This scenario had an additional profit of $\$ 8 /$ cow compared with a similar AI scenario that maximized $\mathrm{NM} \$$, provided that surplus heifer calves could be sold at a premium price based on the superiority of the EBV of NM\$. For the IVP-ET system to be at least as profitable as the comparable AI system during a 15-yr investment period, the surplus calves from the IVP-ET system needed to be sold at the premium prices. The break-even price of fresh embryos was estimated to be $\$ 84$ for the exclusive IVP-ET system. This resulted in the same profit as the AI system, which maximized $\mathrm{NM} \$$ for a 15-yr investment period and in which heifer calves were sold at a premium price.

Key words: stochastic modeling, embryo transfer, genetics, profitability

\section{INTRODUCTION}

In vitro-produced embryo transfer (IVP-ET) systems allow for the production of embryos from genetically superior donors at a young age that can be transferred into recipients in the same or other herds. Genetic progress in the herd can be accelerated because use of an IVP-ET system can increase the intensity of selection, reduce the generation interval, and increase the accuracy of selection (Ruane, 1991; Kruip et al., 1994; Hansen and Block, 2004). The use of an IVP-ET system in a herd to improve the genetics of the herd is growing in popularity (Haag and Dorshorst, 2013; Sjostrom, 2016).

The IVP-ET system increases selection intensity because of the increased number of offspring that can be produced from superior females. In Holsteins, an average of approximately 4 transferable embryos can be produced per collection (Wilson et al., 2006; Bouquet et al., 2015). Oocyte collection can be performed every 2 wk or more frequently as well as into the third month of gestation in pregnant animals (Qi et al., 2013). Even in a conservative IVP-ET system, where oocytes are collected only 7 times/yr from the same female and the calving rate of recipients is 25\% (Pontes et al., 2010), 
it is possible to produce 7 calves/yr per donor compared with only 1 calf from AI. With such a production capacity and the use of sexed semen to produce $90 \%$ females (DeJarnette et al., 2008), it is feasible to use IVP-ET to produce all pregnancies from the best $2 \%$ of the females (heifers and cows) in the herd. In such a system, the use of sexed semen further increases the selection intensity by enabling culling of up to $50 \%$ of the heifer calves born every year (Pryce et al., 2010).

Genomic testing allows the top and bottom of the herd to be identified with greater reliability than traditional EBV based on parent averages (Meuwissen et al., 2001). The EBV are estimates of true breeding values (TBV), which in practice are unknown. Greater reliabilities increase the accuracy of these estimates. Previous modeling studies have found that using genomic EBV to make early selection decisions of sires can increase genetic gains by 30 to $217 \%$ over genetic gain in dairy herds based on parent average selection (Schaeffer, 2006; König et al., 2009). Additional benefits of an IVP-ET system compared with AI systems may include added value of heifer calves not used as replacements, optimization of herd turnover rates, reduced dystocia, and improved biosecurity if open herds could be closed (De Vries et al., 2008; Heikkilä and Peippo, 2012).

One major disadvantage of IVP-ET is the greater cost per breeding and pregnancy compared with AI. For example, Ribeiro et al. (2012) calculated a difference in the cost of a female pregnancy to be $\$ 329$ higher for IVP-ET than for AI using sexed semen. That study did not include genetic and additional benefits from IVPET, however. The increased cost includes the expense of the IVP-ET procedures but also higher probabilities of abortion and neonatal death loss (Taverne et al., 2002; Bonilla et al., 2014). Given the genetic benefits but greater costs, it is not clear how exclusive IVP-ET systems compare with exclusive AI systems regarding genetic, technical, and financial performance. Of interest is how much the genetic changes made through an IVP-ET system affect the technical and financial performance of the IVP-ET system and the break-even cost per transfer to make the IVP-ET system financially competitive with AI. In some related work, Pryce et al. (2010) used a deterministic model and estimated that IVP-ET in the cow-dam selection pathway could result in a yearly genetic gain of $0.59 \mathrm{SD}$ of the genetic trait under selection compared with 0.47 SD when selection occurred without IVP-ET.

Bouquet et al. (2015), working with stochastic simulation and the Viking Red breeding scheme, showed that multiple ovulation and embryo transfer increased genetic gain without increasing inbreeding rates provided that the multiple ovulation and embryo transfer nucleus size and number of sires were large enough.
Increasing the number of flushings per heifer had a greater effect on genetic gain with simultaneous reduction in inbreeding rates compared with increasing the number of flushed heifers. Moreover, genetic gain and reduction in inbreeding rates was greater when more heifers in the population were genotyped to select the heifers that were flushed.

None of these 3 studies (Pryce et al., 2010; Ribeiro et al., 2012; Bouquet et al., 2015) estimated the financial benefits that result from using embryo transfer in combination with genetic selection strategies. Thomasen et al. (2016) reported that the greatest increase in economic value of genetic gain for a 2-trait selection goal was obtained when juvenile IVP-ET was used along with genomic selection in the bull-dam part of the Danish dairy population. This was compared with a scenario without genomic selection and no IVP-ET. Combining IVP-ET with genomic testing was profitable in almost all evaluated scenarios when the cost of producing a calf by IVP-ET ranged from $\$ 500$ to $\$ 1,500$. They did not study the cost of IVP-ET to improve the female performance in a closed herd. We did not find studies that reported the genetic, technical, and financial herd performance from using IVP-ET on just the cow-dam side in a herd.

Earlier we developed a stochastic dynamic model in which the 12 traits included in the lifetime net merit (NM\$) index (VanRaden and Cole, 2014) were modeled simultaneously to study genetic, technical, and financial performance of AI systems over time (Kaniyamattam et al., 2016). In that study we showed that inclusion of multitrait genetic variation and correlations in a model for evaluating reproduction and replacement strategies changed the genetic, technical, and financial results considerably. Further, we showed that the selection focus of surplus heifer calves (e.g., based only on milk yield or NM\$) had significant effects on herd results over time. The model included only AI systems but could be expanded to include IVP-ET systems as well.

The current study had 3 objectives. The first objective was to describe the incorporation of an IVP-ET system into an existing stochastic dynamic model. The second objective was to compare the genetic, technical, and financial performance of exclusive IVP-ET systems with that of exclusive AI systems given realistic prices for embryos and surplus calves. Selection of donors and surplus heifers was either random or based on the EBV of milk yield, daughter pregnancy rate (DPR), or NM\$ in a closed dairy farm. The third objective was to estimate the break-even prices of IVP embryo transfers to obtain the same financial herd performance as an exclusive AI system, depending on how surplus calves were priced. 


\section{MATERIALS AND METHODS}

\section{Brief Description of the Original Model}

For this study we modified the daily dynamic stochastic Monte Carlo model described by Kaniyamattam et al. (2016). In this original model, a herd of individual animals was simulated over time while the herd's genetic, technical, and phenotypic performance was measured. The herd consisted of fetuses (conception to birth), young stock, and cows. Daily phenotypic performance for each animal (e.g., survival, milk yield, and fertility) depended on (1) standard phenotypic functions, such as lactation curves that represented baseline genetics (TBV of traits was 0), and (2) phenotypic deviations from these phenotypic functions, which were the sums of each animal's TBV and permanent and temporary environmental deviations for 12 genetically correlated traits included in the $\mathrm{NM} \$$ index. Reproduction was based on estrus detection and AI in the original model. Eligible females were artificially inseminated by sires that had TBV similar to the predicted genetic trends for Holsteins (VanRaden and Cole, 2014). Sires were independent of the animals in the herd. The TBV of fetuses were based on those from the dam and sire as well as Mendelian sampling. All heifers were ranked by their EBV of the trait of most interest to the farm, such as milk yield, DPR, or NM\$. The lowest ranking surplus heifer calves were sold every month to maintain an approximately $34 \%$ annual cull rate over time. The number of milking cows $(\mathrm{n}=1,000)$ was kept constant over time. If needed, cows ranking lowest for phenotypic milk yield were sold when too many raised heifers were calving. This is referred to as rebalance culling. Genetic and therefore technical changes over time in the herd were the result of genetic trends in the sires and selection of surplus heifer calves and, therefore, genetically improved fetuses, which would become the dams at a later time in the simulation. Technical performance was calculated from outputs such as milk produced, calves sold, number of inseminations, feed intake, and so on. Financial performance was calculated by multiplying technical performance results with relevant prices.

\section{Modeling the IVP-ET System}

Modeling of the IVP-ET system consists of 3 parts: (1) selection of an appropriate ovum pick-up (OPU) protocol, (2) selection of donors, and (3) selection of recipients.

OPU Protocol. We surveyed various OPU protocols reported in the literature and relied on the authors' expertise in choosing a protocol that is currently used commercially. In our chosen protocol, donors selected for each day were assumed to undergo removal of a dominant follicle on the same day they were selected. After an interval of $36 \mathrm{~h}$, each donor received 6 injections of FSH at 12-h intervals (Nivet et al., 2012). The OPU occurred $48 \mathrm{~h}$ after the last injection of $\mathrm{FSH}$ (Chaubal et al., 2006). A deterministic number of 8 oocytes was assumed to be collected from each donor at each OPU. Oocytes retrieved were matured for 24 $\mathrm{h}$ and were assumed to be fertilized with sexed semen. The probability that an embryo was female was set at $85 \%$. We also assumed that the probability that an oocyte became a transferable embryo was $53 \%$ (Nivet et al., 2012). Hence, on average, 4.25 transferable embryos were produced per OPU (Vieira et al., 2014; Bouquet et al., 2015). We assumed that transferable embryo production was the same for cows and heifers (Rizos et al., 2005). Both embryo sex and transferability were determined stochastically per oocyte.

Selection of Donors. We modeled exclusive IVPET systems in which all pregnancies were obtained from ET and exclusive AI systems in which all pregnancies were obtained through AI. For a herd of 1,000 milking cows and associated young stock, approximately 1,000 and 375 conceptions in cows and heifers, respectively, were needed annually for AI systems (Kaniyamattam et al., 2016). In the IVP-ET systems, approximately 2,400 embryos were required for cows and 700 embryos were required for heifers to achieve these conceptions. With an average of 4.25 transferable embryos per OPU, it was necessary to collect oocytes from 14 donors/wk. Because we did not synchronize the recipients in our model, we needed fresh transferable embryos every day to impregnate the animals in the herd coming into estrus daily. Therefore, oocytes were collected from 2 donors daily.

To select donors, a list of eligible females was created weekly. Both heifers and cows were eligible to be embryo donors. Eligible females were ranked by the EBV of the trait of interest such as milk yield or NM\$ (explained later), and the top 14 females were selected to be donors for a week. Females that were donors one week were not eligible to be donors again the following week. In addition, a heifer donor could be collected for a maximum of 4 times between 11 mo of age and the end of the heifer voluntary waiting period at $400 \mathrm{~d}$ of age. Once a heifer was confirmed pregnant at $42 \mathrm{~d}$ after insemination, she was again eligible to be a donor up until $90 \mathrm{~d}$ of pregnancy (maximum of 3 collections). This constraint on 7 collections was implemented to prevent culling of genetically superior heifers from the herd because of nonpregnancy. For cow donors, collections happened in nonpregnant cows from d 50 to 
d 70 after calving (maximum of 2 collections) and in pregnant cows from 42 to $90 \mathrm{~d}$ of gestation (maximum of 3 collections).

The OPU protocol was initiated for 2 donors on each day of the week so that approximately 8.5 transferable embryos were available for transfer each day. Embryos harvested at d 7 after collection were transferred to recipients on d 6,7 , or 8 of their estrous cycle. Surplus embryos that were not transferred at the end of the day were frozen.

Selection of Recipients. Heifers and cows that were not donors, were not pregnant, and were past their voluntary waiting period were eligible recipients. Eligible recipients were reverse ranked for the trait of interest so that the lowest ranked animals had the first chance to receive a randomly chosen embryo. In general, all eligible recipients received embryos. Once estrus was observed in an eligible recipient, the recipient was scheduled to receive an embryo on d 6,7 , or 8 after estrus whenever a fresh embryo was first available. Frozen embryos were transferred in the unlikely event that fresh embryos were not available.

\section{Fertility of Recipients and AI}

The standard phenotypic probability of conception for a transferred fresh or frozen embryo, as well as an AI, was set at $35 \%$ in cows. In heifers, the standard phenotypic probability of conception for fresh and frozen embryos was $60 \%$ for first transfer as well as for first AI, with a 5 percentage point reduction for every next transfer or insemination with a minimum of $45 \%$. These probabilities represented the likelihood of pregnancy on d 35 after insemination or collection of oocytes from the donor as determined by pregnancy diagnosis on that day. The actual individual probability of conception depended on the sum of the genetic and environmental deviations of the traits DPR and cow conception rate (Kaniyamattam et al., 2016). Due to selection within the herd and changes in fertility traits from the sires, the average probability of conception changed with time.

The first $2 \mathrm{AI}$ in the top $50 \%$ of heifers were conducted with sexed semen depending on selection criteria as described later. The probability of conception on d 35 after use of sexed semen was reduced by $15 \%$ compared with conventional AI (Seidel, 2007).

The risk of embryonic mortality before d 35 was set at 0 because it was included in the probability of conception. The daily abortion risk after d 35 from IVP-ET was estimated from a logarithmic curve that resulted in $22.5 \%$ abortions in cows and $15 \%$ abortions in heifers, with $50 \%$ of abortions happening between d 35 and d 45 of conception. The daily abortion risk in cows and heifers after AI was estimated from a logarithmic curve, which resulted in 10\% abortions in cows and 5\% abortions in heifers. This is similar to the cumulative risk of abortion used in Kaniyamattam et al. (2016). The stillbirth risk was $6 \%$ for IVP-ET calves (Bonilla et al., 2014) and $3 \%$ for calves conceived from AI. Estrus detection, conception, and abortion were triggered stochastically.

\section{Experimental Design}

A starting herd with only AI was simulated once for 10 yr (d $-5,475$ to $d-1,875)$ to obtain a steadystate herd as described in Kaniyamattam et al. (2016). Randomly chosen surplus heifers aged 3 to 4 mo were sold every month to maintain an approximately $34 \%$ annual cow cull rate. The next 5 yr (d $-1,874$ to $d 0)$, the simulation was repeated 100 times with the same AI-only strategy to obtain average herd statistics with small standard errors. At the end of d 0, the herd's genetic and technical results resembled a plausible U.S. dairy herd in 2015.

Eight scenarios (Table 1) were simulated for the next 15 yr (d 1 to d 5,475), starting with the same herd saved at the end of $d 0$. These 8 scenarios were a combination of reproduction system (IVP-ET or AI) and selection criteria (EBV of milk, DPR, NM\$, or random selection) for selection of donors and recipients (in the case of IVP-ET), identification of the top $50 \%$ of heifers to inseminate with sexed semen (in the case of AI), and identification of which heifer calves to sell as surplus. Selection on 1 of 3 traits (milk, DPR, $\mathrm{NM} \$$ ) or random selection represented different genetic selection focuses in the female selection pathway. The 8 scenarios allowed for estimation of short-term and long-term genetic, technical, and financial effects of using an exclusive IVP-ET system versus a comparable exclusive AI system.

Genomic testing was used after d 0 on all heifer calves at $90 \mathrm{~d}$ of age, which increased the reliabilities of the EBV of the 12 genetic traits and hence improved the accuracy of identification of animals to be donors, recipients, or sold. The genetic trends of the sire traits were the same in all 8 scenarios and mimicked the expected genetic trend in $\mathrm{NM} \$$ over time (VanRaden and Cole, 2014).

The AIRAND scenario continued the AI strategy from the first $15 \mathrm{yr}$. Heifers receiving sexed semen and surplus heifers to be culled were selected randomly. The ETRAND scenario used IVP-ET, but donors, recipients, and surplus heifers were selected randomly. In the AIMILK, AIDPR, and AINM scenarios, the top $50 \%$ of heifers to be inseminated with sexed semen were selected from the highest ranking eligible heifers 
Table 1. Rank criteria and sexed semen use in 8 scenarios to illustrate the model and to test different AI and embryo transfer systems

\begin{tabular}{lll}
\hline Scenario $^{1}$ & Rank criterion & Sexed semen use \\
\hline AIRAND & Random & Random $50 \%$ of heifers \\
AINM & EBV of NM $\$$ & Top 50\% of heifers \\
AIMILK & EBV of milk & Top 50\% of heifers \\
AIDPR & EBV of DPR & Top 50\% of heifers \\
ETRAND & Random & Oocytes collected from randomly selected $2 \%$ of donors \\
ETNM & EBV of NM $\$$ & Oocytes collected from top $2 \%$ of donors \\
ETMILK & EBV of milk & Oocytes collected from top $2 \%$ of donors \\
ETDPR & EBV of DPR & Oocytes collected from top $2 \%$ of donors \\
\hline
\end{tabular}

${ }^{1} \mathrm{AI}=$ exclusive AI system; ET = exclusive in vitro-produced embryo transfer (IVP-ET) system. Rank criteria used in each scenario for culling of surplus heifers and selection of heifers or oocyte donors. Eligible animals were ranked either randomly (RAND) or based on the EBV of net merit (NM), milk yield (MILK), or daughter pregnancy rate (DPR). In the AI scenarios, the top 50\% of heifers received sexed semen in the first 2 inseminations. In the IVP-ET scenarios, the top $2 \%$ of dams was selected to be donors. Half of the selected dams produced oocytes in $1 \mathrm{wk}$, whereas the other half was not involved in collection that week. The oocytes were fertilized with sexed semen.

for EBV of milk yield, DPR, or NM\$, respectively. Surplus heifer calves were sold from the lowest ranking heifer calves for these traits. In the ETMILK, ETDPR, and ETNM scenarios, the donors were selected from the highest ranking eligible females for EBV of milk yield, DPR, or NM\$, whereas the recipients were selected from the lowest ranking eligible recipients for these traits. Surplus heifer calves were also sold from the lowest ranking heifer calves.

\section{Input Prices}

Default input prices and costs used in the model are described in Table 2 based on data in Kaniyamattam et al. (2016). The cost of an IVP-ET was set at $\$ 165$ from our experiences with IVP-ET services. The $\$ 165$ is a per-embryo price that includes the production of the embryo (i.e., OPU and IVF) and the cost of transfer of the embryo. We assumed that embryos were transferred by on-farm personnel with sufficient training and that labor was not different from the labor involved with AI. Frozen embryos cost $\$ 30$ more than fresh embryos. When frozen embryos were available at the end of each year, we assumed that they were sold at $\$ 195 /$ embryo. Bull calves were sold at birth.

In one set of analyses, 3- to 4-mo-old surplus heifer calves were sold at a fixed price of $\$ 500$. The genetic merit (e.g., EBV of NM\$) of the sold heifer calves varied greatly between scenarios, however. Therefore, we also evaluated the sale of surplus heifer calves born from the IVP-ET system at a premium price that reflected their genetic merit in addition to the $\$ 500$ base price. The premium price was calculated as the net present value of the difference in average $\mathrm{EBV}$ of $\mathrm{NM} \$$ of heifer calves sold in the same month from the IVP-ET and AI scenarios with the same selection focus. We assumed that $85 \%$ of the kept heifer calves became cows and that the average expression of the genetic differences occurred $3.5 \mathrm{yr}$ after birth. The discount rate was set at 5\%. For example, if the $\mathrm{NM} \$$ of sold heifer calves from the IVP-ET system was on average $\$ 100$ greater than the $\mathrm{NM} \$$ of the sold heifer calves from the AI system, then the premium per sold IVP-ET heifer calf was calculated as $\$ 100 \times 0.85 \times(1 / 1.05)^{3.5}=\$ 72$.

Table 2. Prices $(\$)$ used for calculating revenues and costs in the simulation model

\begin{tabular}{lc}
\hline Item & Value \\
\hline Milk price/kg & 0.07 \\
Fat price/kg & 4.30 \\
Protein price/kg & 5.47 \\
SCC premium or penalty ${ }^{1}$ & 0.0019 \\
Cull cow value/head & 800.00 \\
Sold bull calf base value/head & 150.00 \\
Sold heifer calf base value/head ${ }^{2}$ & 500.00 \\
Feed price/kg of DM (lactating cow) & 0.30 \\
Feed price/kg of DM (dry cow) & 0.20 \\
Heat detection labor/eligible day & 0.05 \\
Conventional semen insemination & 15.00 \\
Sexed semen insemination & 30.00 \\
Fresh embryo production and transfer & 165.00 \\
Frozen embryo production and transfer & 195.00 \\
Frozen embryo sale price & 195.00 \\
Pregnancy diagnosis & 5.00 \\
Genomic testing & 40.00 \\
Other variable costs/cow per day & 0.50 \\
Fixed costs/stall per day & 2.00 \\
Purchased replacement heifer cost & $2,000.00$ \\
Heifer calf raising cost/day ( $\leq 55$ d of age) & 4.80 \\
Heifer calf raising cost/day (>55 d of age) & 2.00 \\
Cull price/kg of BW & 2.00 \\
\hline
\end{tabular}

${ }^{1}$ Premium or penalty per $100,000 \mathrm{SCC} / \mathrm{kg}$ of milk below (premium) or above (penalty) 200,000 SCC.

${ }^{2}$ The difference in EBV of net merit of surplus heifer calves from similar in vitro-produced embryo transfer and AI scenarios was used to determine the premium price of the sold heifer calf value. The sale price is at $105 \mathrm{~d}$ of age for heifer calves. The cost of raising the heifer to $105 \mathrm{~d}$ is accounted for separately. 


\section{Replicates and Statistical Analysis}

Each scenario was replicated 100 times to generate standard errors of the means as small as feasible given the speed of the Java computer code of the model and the available hardware. The GLM procedure in SAS version 9.4 (SAS Institute Inc., Cary, NC) was used for one-way ANOVA of output statistics among the scenarios.

\section{RESULTS}

Table 3 shows the average TBV of 13 traits, including $\mathrm{NM} \$$, for cows in the herd for yr 0 at the start of the simulation of the 8 scenarios. The average TBV of NM\$ was $-\$ 4$, which was close to the target of $\$ 0$. Thus, the 8 scenarios were simulated from a starting herd with a $21 \%$ pregnancy rate, $145 \mathrm{~d}$ open, and $10,679 \mathrm{~kg}$ of milk/cow per year. The annual cull rate was $31 \%$, and $8 \%$ of the heifer calves born on the farm were sold as surplus calves.

Figures 1, 2, and 3 show the increases in the average TBV of the traits NM $\$$, milk, and DPR of sires and cows during the last $20 \mathrm{yr}$ (yr -4 to 15) of simulation for the 8 scenarios. Generally, the genetic lag between the sire and average cow TBV decreased after yr 3 and stabilized by yr 15 as a result of genetic selection for the trait of interest within the herd. Table 3 also shows the genetic and technical results in only yr 15 for the 8 scenarios. Genetics of all traits and technical performances had changed in the desired direction by yr 15 in both the AI and IVP-ET systems but at different rates.

\section{Genetic and Technical Performance of the Al Systems}

The average change in cow TBV of $\mathrm{NM} \$$ in yr 15 from yr 0 was $\$ 2,136$ for the AIRAND scenario, which was solely attributable to an increase in the TBV of $\mathrm{NM} \$$ of the sires (Table 3, Figure 1). For all other traits (except the 3 fertility traits, milk yield, and protein yield), the greatest change in TBV was accomplished with the AINM scenario. The increase in TBV of NM\$ of cows was the greatest for the AINM scenario from yr 3 to yr 15, as expected. The AIMILK scenario resulted in the greatest TBV for milk $(2,159 \mathrm{~kg})$ and protein $(71$ $\mathrm{kg}$ ) and the highest phenotypic milk yield of $13,902 \mathrm{~kg} /$ cow per year. Because the TBV of milk is negatively correlated with the TBV of the 3 fertility traits, this scenario resulted in the lowest average TBV for DPR (Figure 3) and heifer conception rate and cow conception rate (not shown) across the 15 yr. Consequently, the phenotypic pregnancy rate in yr 15 (33\%) was lower for AIMILK than for the other 3 AI scenarios, including random selection in the AIRAND scenario. In contrast, the TBV of the 3 fertility traits were the greatest in the AIDPR scenario, which resulted in a $37 \%$ pregnancy rate and subsequently the lowest days open of $99 \mathrm{~d}$. The number of surplus heifer calves was smaller when the TBV of the 3 fertility traits was the smallest. The age of the dam at creation of her embryo decreased from 1,080 to $941 \mathrm{~d}$ (Table 3) during the $15 \mathrm{yr}$ in the AINM scenario, whereas the other $3 \mathrm{AI}$ scenarios resulted in slightly older dams at creation of the embryo.

\section{Genetic and Technical Performance of the IVP-ET Systems}

The ETRAND scenario resulted in $\$ 166$ greater TBV of NM $\$$ of the cows in the herd by yr 15 than the AIRAND scenario (Table 3 ) because of the use of younger animals as donors of embryos, which decreased the genetic lag with the sires. The TBV of $\mathrm{NM} \$$ was maximized with the ETNM scenario $(\$ 2,983)$ in yr 15 among all 8 scenarios. Moreover, this scenario resulted in the greatest desired change in TBV of all traits except for milk, protein, and the 3 fertility traits in yr 15 . Because the TBV of productive life was also greatest with the ETNM scenario ( $25 \mathrm{mo}$ ), it led to the smallest involuntary cull rate of $14 \%$ and the greatest rebalance cull rate of $22 \%$. A reduction of $586 \mathrm{~d}$ in age of dam at creation of her embryo occurred between ETNM (355 d) and AINM (941 d). This reduction is attributable to donors being young heifers compared with cows, which produce the majority of the embryos in the AI scenarios.

The ETMILK scenario led to the greatest TBV of milk and phenotypic milk yield among the 8 scenarios. The genetic lag in TBV of milk in yr 15 between the ETMILK and ETDPR scenarios was $1,176 \mathrm{~kg} / 305 \mathrm{~d}$. The ETMILK scenario also led to the lowest cow TBV of the 3 fertility traits and phenotypic pregnancy rate (31\%) among the ET scenarios because of the negative genetic correlations between milk and fertility. The lower phenotypic pregnancy rate resulted in a lower percentage of surplus heifer calves sold $(53 \%)$. The average TBV of all 3 fertility traits in the ETMILK scenario were much lower than in the AIMILK scenario. The average TBV of milk of the cows was greater than that of the sire after yr 10 (Figure 2).

Selection for DPR in the ETDPR scenario rapidly increased the TBV of DPR of the cows after yr 3 and surpassed the TBV of DPR of the sire in yr 5 and later. After yr 5 the increase became smaller, and the annual increase was similar to that of the sire by yr 15 (Figure 3 ). In yr 15, the TBV of DPR of the cows was 4.29 percentage points greater than the TBV of DPR of the 
EMBRYO TRANSFER VERSUS ARTIFICIAL INSEMINATION

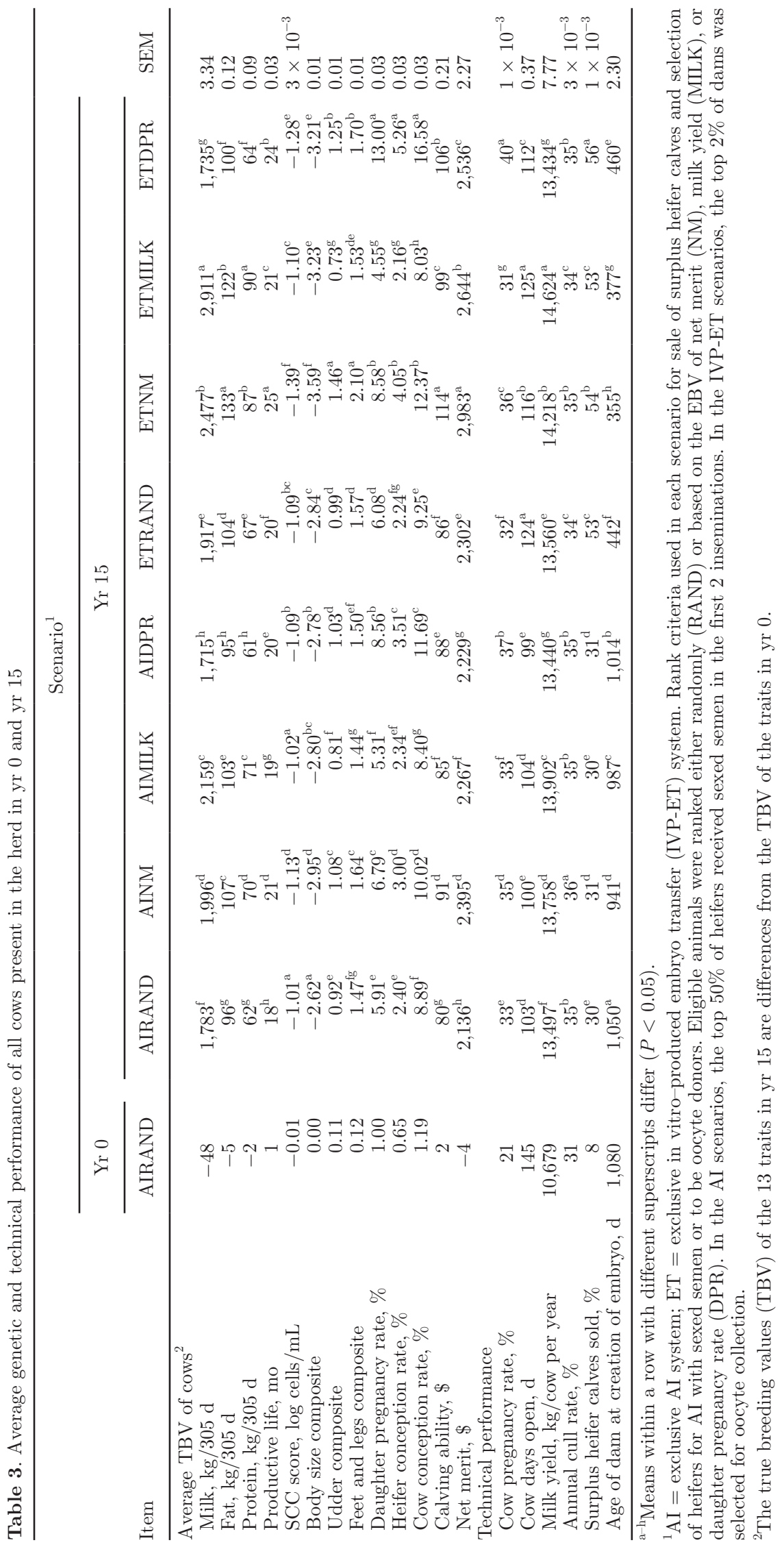


sire in the ETDPR scenario. The TBV of DPR of the cows was 8.45 percentage points greater for the ETDPR scenario than for the ETMILK scenario in yr 15. The phenotypic cow pregnancy rate was the greatest among the 8 scenarios. Cow days open was the smallest among the 4 IVP-ET scenarios. Cow days open were greater for the IVP-ET scenarios than for the AI scenarios because cows received embryos approximately 1 wk after estrus and had greater risk of abortion.

Table 4 shows donor and recipient statistics in yr 15 for the 4 IVP-ET scenarios. The number of transferable embryos was similar across all 4 scenarios because the number of oocyte collections in 1 yr (728) was fixed in the model. The ETDPR scenario resulted in more cow donors (162) than the other 3 IVP-ET scenarios. In this scenario, the TBV of DPR of cows in yr 15 and heifers were similar due to continuous selection for greater DPR and because the TBV of DPR of the sire was lower than that of the females. Selection for DPR led to the greatest embryo conception rates as a result of the genetically correlated TBV of DPR, cow conception rate, and heifer conception rates. As the phenotypic pregnancy rate increased over time, fewer embryos were needed to establish the same number of pregnancies.
Consequently, the number of embryos that were frozen and left in inventory increased.

\section{Financial Performance of the Al and IVP-ET Systems}

The herd financial performance depended on whether heifer calves were sold at a price that reflected their EBV compared with a fixed base price, which ignored the genetic merit of the calves. Figure 4 shows the changes in the EBV of the sold surplus heifer calves over time for the 8 scenarios. These EBV are the result of the genetic merit of the (donor) mother as well as the number of heifer calves born and hence the fraction surplus calves. The EBV of NM\$ of surplus heifer calves from the ETNM scenario was on average $\$ 557$ greater than the EBV of the surplus heifer calves from the AINM scenario after yr 5, which meant that the premium per sold heifer calf was $\$ 401$.

Figure 5 shows the annual profit per cow per year for the 8 scenarios over time, assuming premium pricing of surplus heifer calves. Figure 5 shows a large decrease in profitability averaging $\$ 369 /$ cow per year in yr 1 to 5 for the ETNM scenario compared with the AINM scenario because of the ET cost. Larger differences are

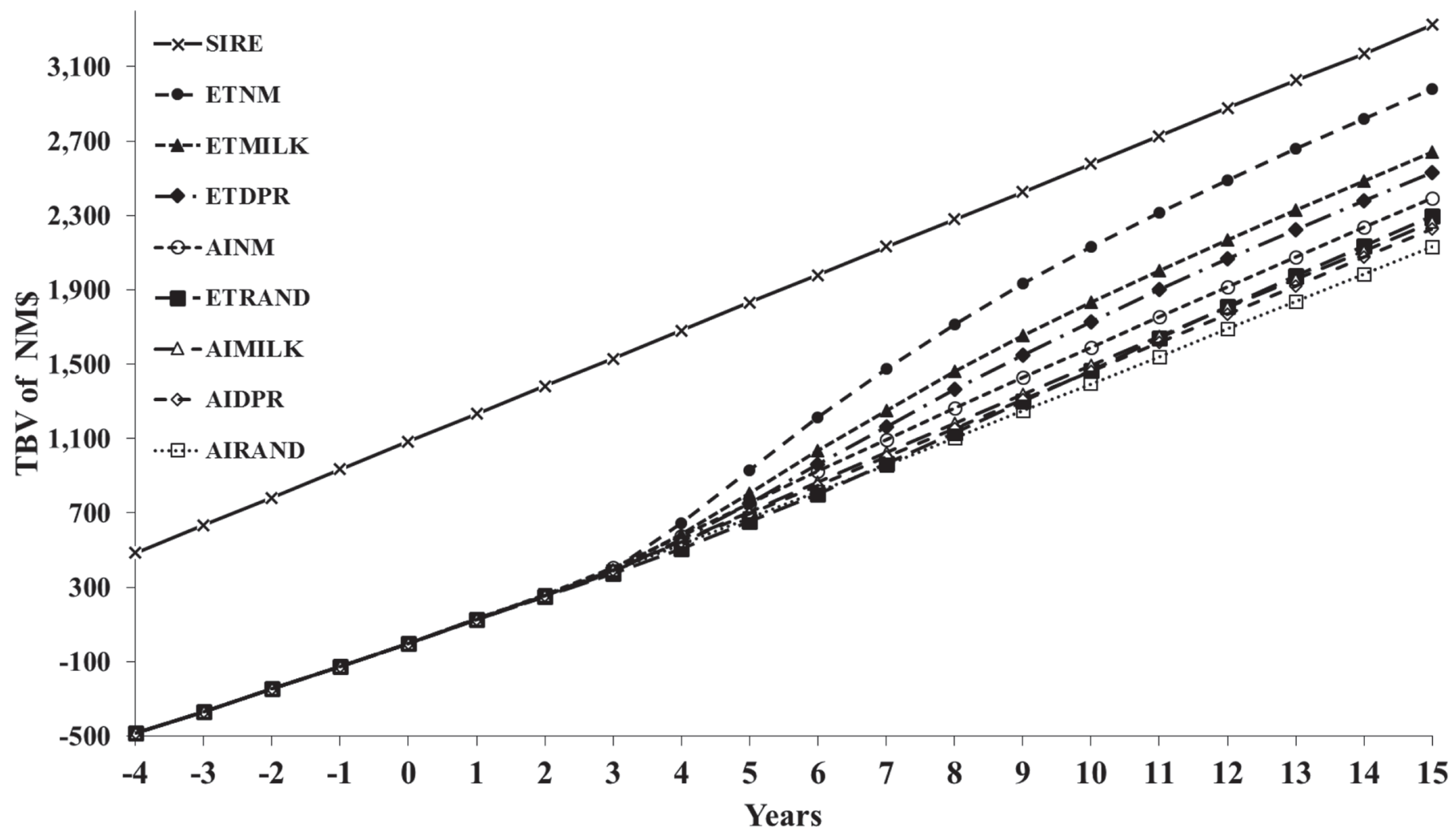

Figure 1. Average true breeding values $(\mathrm{TBV})$ of net merit $(\mathrm{NM})$ in sires and cows from yr -4 to +15 for 8 scenarios. AI $=$ exclusive AI system; ET = exclusive in vitro-produced embryo transfer system. Eligible animals were ranked either randomly (RAND) or based on the EBV of NM, milk yield (MILK), or daughter pregnancy rate (DPR). 


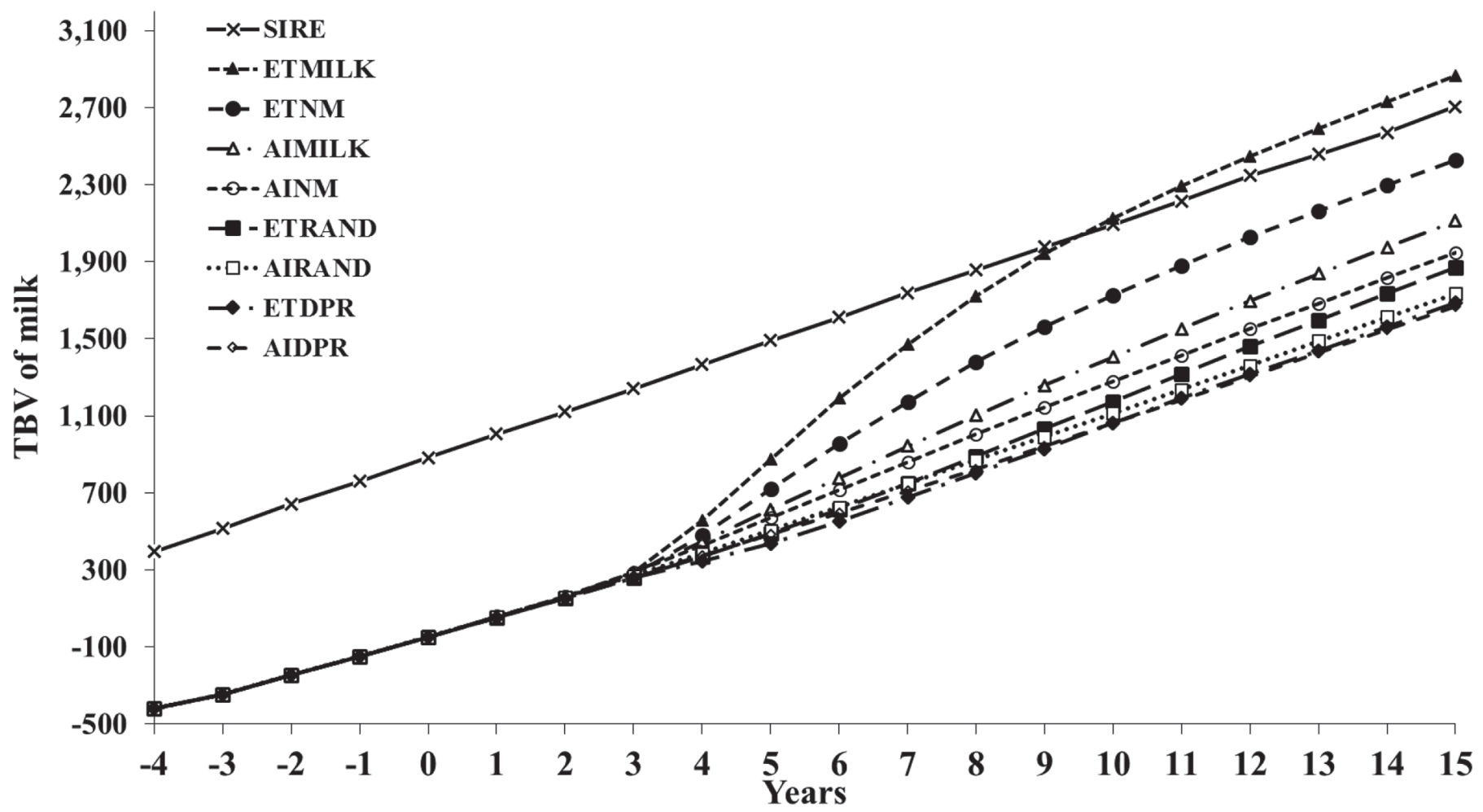

Figure 2. Average true breeding values (TBV) of milk in sires and cows from yr -4 to +15 for 8 scenarios. AI $=$ exclusive $\mathrm{AI}$ system; ET $=$ exclusive in vitro-produced embryo transfer system. Eligible animals were ranked either randomly (RAND) or based on the EBV of net merit (NM), milk yield (MILK), or daughter pregnancy rate (DPR).

observed for the 3 other IVP-ET scenarios. The technical and financial results from the genetically superior replacement heifers start to be realized from yr 3 on when the first IVP-ET produced females start milk production in the herd. Figure 5 also shows that the ETNM scenario takes at least 13 yr for profit per cow per year to be even with the AINM scenario if embryo cost is $\$ 165 /$ transfer. The scenarios ETMILK, ETDPR, and ETRAND result in lower profit per cow per year than their equivalent $\mathrm{AI}$ scenarios.

Table 5 shows financial results of the 8 scenarios in yr 15 as well as for the starting herd in yr 0 . The most profitable scenarios were those in which $\mathrm{NM} \$$ was the trait of selection. The ETNM scenario had the greatest revenues among the 8 scenarios $(\$ 6,427 /$ cow per year); this was $\$ 346 /$ cow per year greater than the AINM scenario when surplus heifer calves were sold at the base price. Even though there was a difference of 481 $\mathrm{kg} / 305 \mathrm{~d}$ in the TBV of milk yield between the ETNM and AINM scenarios (Table 3), there was a difference of only $\$ 233 /$ cow per year in milk sales due to component pricing (Table 5). Breeding costs were on average $\$ 359$ / cow per year greater for the IVP-ET scenarios than for the AI scenarios. Heifer raising cost was also in general greater for the IVP-ET scenarios because more heifer calves were born per year due to the use of sexed semen to fertilize embryos. These heifers were raised on the farm until the genotyping results were available at 3 to 4 mo of age, after which the surplus heifer calves were sold. The greater heifer raising cost was offset by greater calf sales, however. Genomic testing costs consequently were also greater for the IVP-ET scenarios than for the AI scenarios.

Pricing of the surplus calves determined which scenario was most profitable. When surplus calves were sold at a base price, the profits per cow per year when TBV of NM\$, milk yield, or DPR were maximized were $\$ 185, \$ 240$, and $\$ 293$ greater, respectively, for the AI systems compared with the IVP-ET systems in yr 15 (Table 5). When surplus heifer calves were sold at premium prices reflecting their genetic superiority, the profit per cow per year of the IVP-ET systems was greater than that of the AI systems by $\$ 8,-\$ 120$ (a loss), and $-\$ 194$ (a loss) for scenarios that maximized TBV of NM\$, milk yield, or DPR in yr 15 .

Yr 15 Embryo Transfer Break-Even Prices. When premium pricing for heifer calves was assumed, the price of a fresh embryo at which the profit per cow per year in the ETNM scenario broke even with that from the AINM scenario in yr 15 was $\$ 168$ ( $\$ 3$ greater 


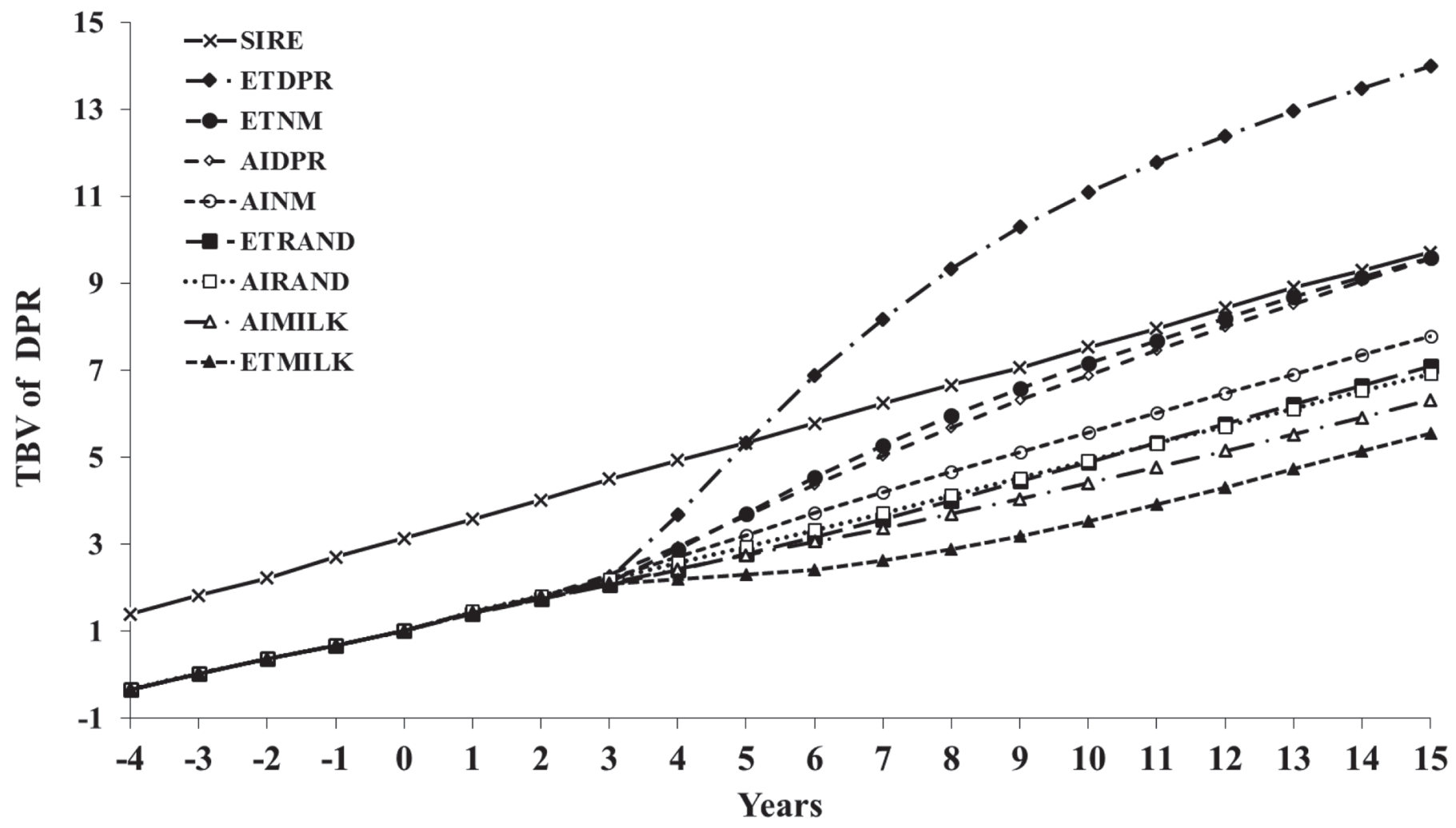

Figure 3. Average true breeding values (TBV) of daughter pregnancy rate (DPR) in sires and cows from yr -4 to +15 for 8 scenarios. AI $=$ exclusive AI system; ET = exclusive in vitro-produced embryo transfer system. Eligible animals were ranked either randomly (RAND) or based on the EBV of net merit (NM), milk yield (MILK), or DPR.

Table 4. Donor and recipient statistics from yr 15 of simulation in 4 in vitro-produced embryo transfer (IVPET) scenarios ${ }^{1}$

\begin{tabular}{|c|c|c|c|c|}
\hline Statistics & ETRAND & ETNM & ETMILK & ETDPR \\
\hline \multicolumn{5}{|l|}{ Donor statistics } \\
\hline Collections from heifers, no. & 674 & 719 & 677 & 566 \\
\hline Oocytes produced from heifers, no. & 5,392 & 5,768 & 5,424 & 4,540 \\
\hline Collections from cows, no. & 54 & 9 & 51 & 162 \\
\hline Oocytes produced from cows, no. & 443 & 68 & 412 & 1,295 \\
\hline Total transferable embryos, no. & 3,312 & 3,315 & 3,323 & 3,329 \\
\hline \multicolumn{5}{|l|}{ Recipient statistics } \\
\hline Eligible heifer recipients, no. & 1,717 & 1,690 & 1,722 & 1,676 \\
\hline Average d 6,7 , and 8 conception rate of heifers, $\%$ & 52 & 53 & 51 & 55 \\
\hline Total fresh transfers in heifers, no. & 802 & 800 & 798 & 801 \\
\hline Frozen d 8 conception rate of heifers, $\%$ & 54 & 52 & 54 & 54 \\
\hline Total frozen transfers in heifers, no. & 35 & 23 & 37 & 16 \\
\hline Total conceptions in heifers, no. & 454 & 458 & 453 & 462 \\
\hline Eligible cow recipients, no. & 4,418 & 4,122 & 4,578 & 3,777 \\
\hline Average d 6,7 , and 8 conception rate of cows, $\%$ & 53 & 58 & 51 & 66 \\
\hline Total fresh transfers in cows, no. & 1,954 & 1,859 & 2,007 & 1,723 \\
\hline Frozen d 8 conception rate of cows, $\%$ & 54 & 58 & 51 & 68 \\
\hline Total frozen transfers in cows, no. & 155 & 102 & 170 & 64 \\
\hline Total conceptions in cows, no. & 1,149 & 1,164 & 1,126 & 1,199 \\
\hline Embryos left in inventory, no. & 366 & 530 & 310 & 726 \\
\hline
\end{tabular}

${ }^{1} \mathrm{ET}=$ exclusive IVP-ET system. Rank criteria used in each scenario for sale of surplus heifer calves and selection of heifers for AI with sexed semen or to be oocyte donors. Eligible animals were ranked either randomly (RAND) or based on the EBV of net merit (NM), milk yield (MILK), or daughter pregnancy rate (DPR). 
than the $\$ 165$ default embryo price in the model; Table $5)$. If the goal was to maximize the TBV of milk or DPR in the herd, the break-even fresh embryo prices were $\$ 120$ and $\$ 80$, respectively, to break even with the respective AI scenarios in yr 15 . When surplus heifer calves were sold at the $\$ 500$ base price, the embryo break-even prices in all IVP-ET scenarios ranged from $\$ 26$ to $\$ 89$ and were lower than the default embryo price of $\$ 165$.

Net Present Value of Cumulative Profitability Differences. Figure 6 shows the net present value of the cumulative difference in profitability between the AINM and ETNM scenarios for 5 embryo transfer prices at a $5 \%$ annual discount rate. Premium pricing of surplus heifer calves was assumed. A positive value implies that the IVP-ET system has yielded more profit than the comparable AI system from yr 0 until the year investigated. Embryo transfer prices of $\$ 100$ or less showed an initial increase in net loss of the IVPET systems compared with the AI systems, but after several years the net loss decreased. It turned into a net gain within $15 \mathrm{yr}$ (when embryo prices were $\$ 84$ or lower) or later (when embryo price was above $\$ 84$ ) when the value of improved genetics was being realized.
The $\$ 150$ and $\$ 200$ embryo price showed a continuously increasing net loss for the IVP-ET system.

When the cost of a fresh embryo was $\$ 200$, the investment in IVP-ET resulted in an average loss of $\$ 222$ /cow per year, or $\$ 3,330 /$ cow in the first 15 yr. If the cost of a fresh embryo was $\$ 150$, then the average loss from the ETNM system decreased to $\$ 126 /$ cow per year. At a cost of $\$ 100 /$ fresh embryo, the cumulative net loss from the ETNM system over the AINM system decreased to $\$ 31 /$ cow per year by the end of yr 15 . If the cost of a fresh embryo was $\$ 50$, then the average net gain from the ETNM system over the AINM system was $\$ 65 /$ cow per year during the 15-yr investment period.

The break-even price of a fresh embryo was $\$ 84$ when the exclusive IVP-ET system was as equally profitable as the AI system that maximized $\mathrm{NM} \$$ for a $15-\mathrm{yr}$ investment period and sold calves at a premium price. Break-even embryo prices for the 10- and 5-yr investment period were $\$ 62$ and $\$ 22$, respectively. The ETNM scenario became more profitable than the AINM scenario in yr 13 if the fresh embryos were provided at the default price of $\$ 165$ (Figure 5).

If the surplus heifer calves were sold at the $\$ 500$ base price, the break-even embryo price was $\$ 35$ for

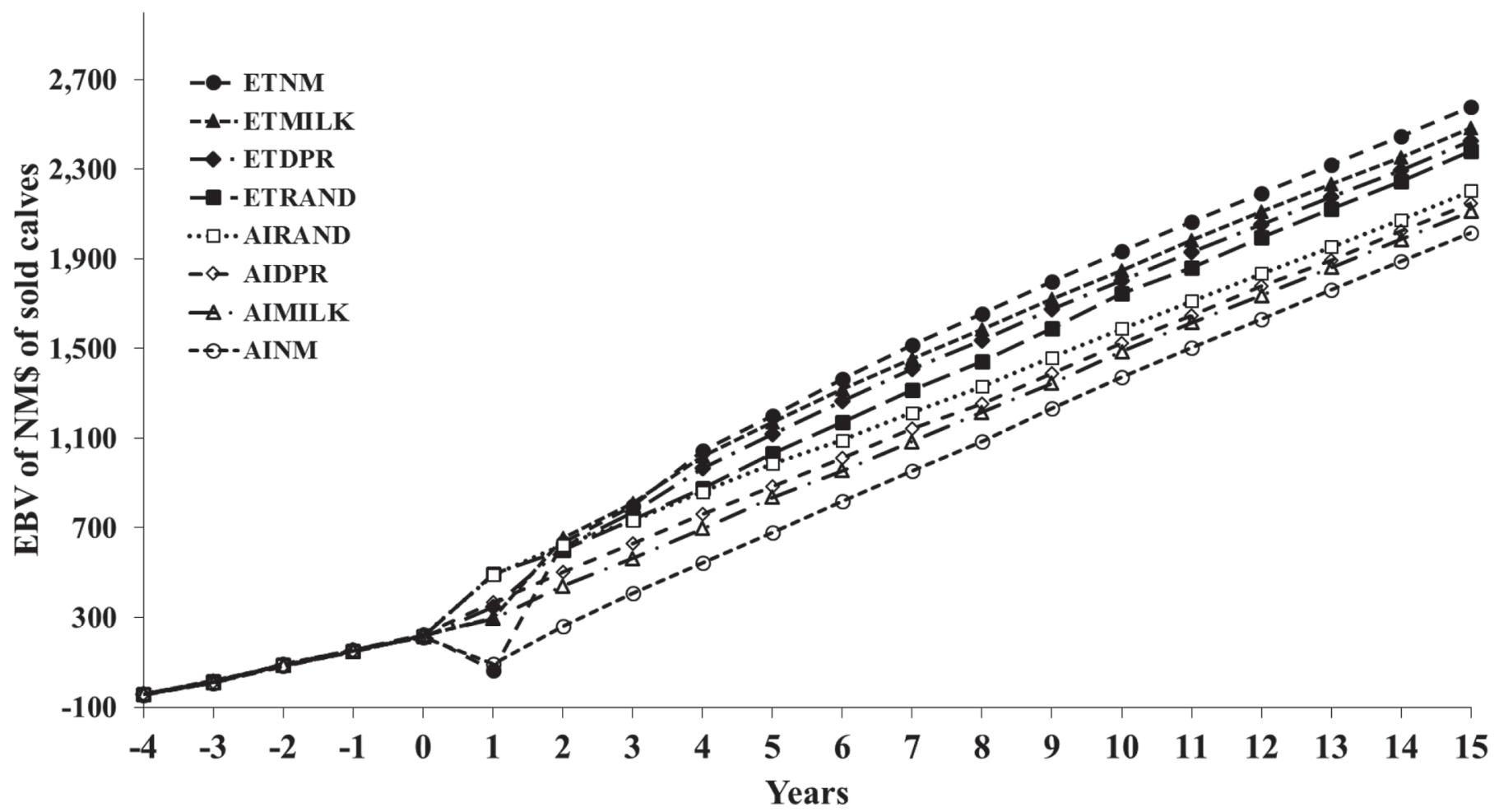

Figure 4. Average EBV of net merit (NM) of surplus heifer calves sold from yr -4 to +15 . AI $=$ exclusive AI system; ET $=$ exclusive in vitro-produced embryo transfer system. Eligible animals were ranked either randomly (RAND) or based on the EBV of NM, milk yield (MILK), or daughter pregnancy rate (DPR). 


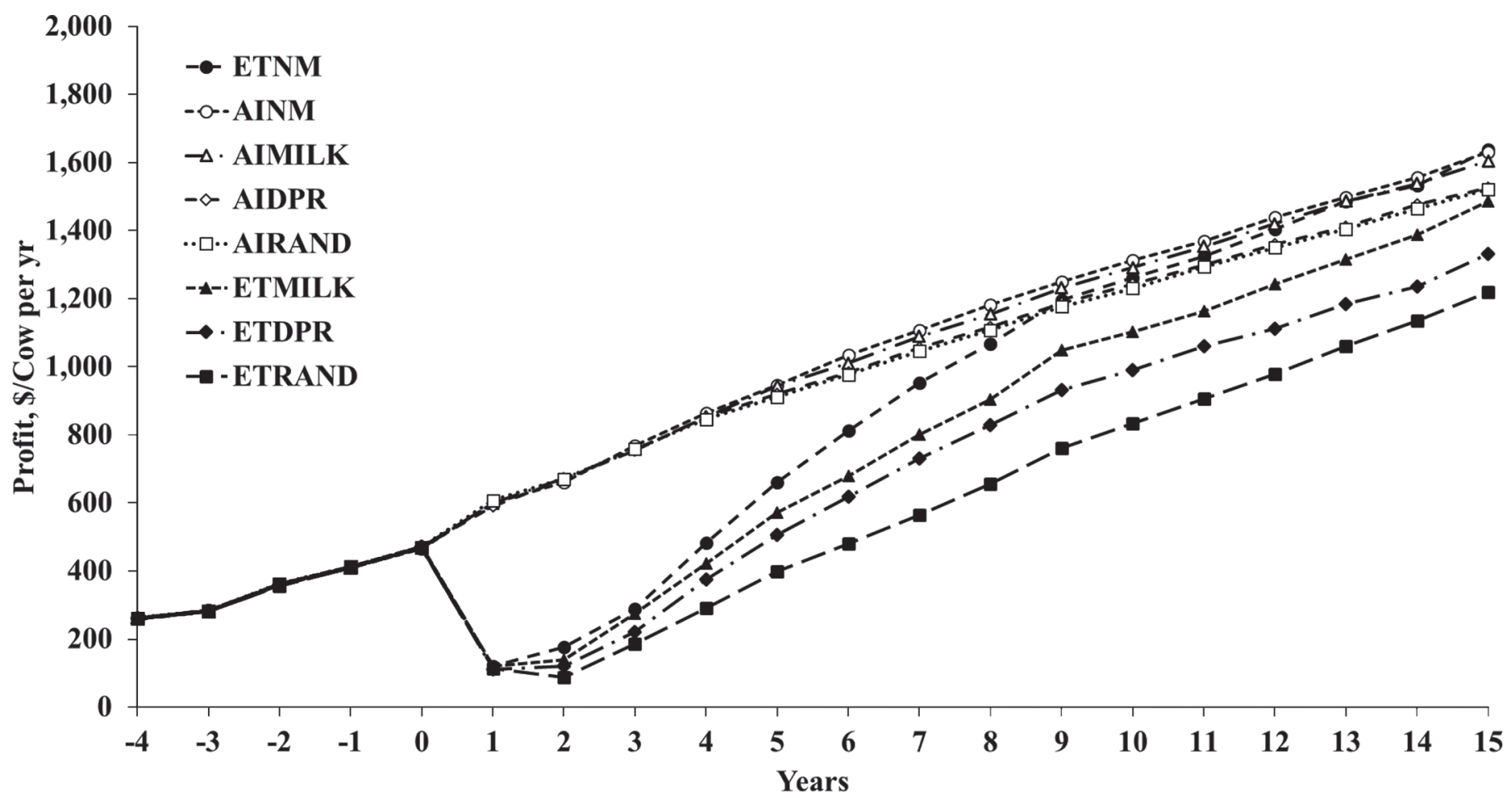

Figure 5. Profit per cow per year from yr -4 to +15 for 8 scenarios. Premium pricing of surplus heifer calves is assumed. AI $=$ exclusive AI system; ET = exclusive in vitro-produced embryo transfer (IVP-ET) system. Eligible animals were ranked either randomly (RAND) or based on the EBV of net merit (NM), milk yield (MILK), or daughter pregnancy rate (DPR). The profit of the 4 IVP-ET scenarios decreases rapidly after the start of the IVP-ET system in yr 1 because the embryo transfer prices are greater than the AI prices.

the ETNM scenario to be as equally profitable as the AINM scenario for the 15-yr investment period. Breakeven embryo prices for the 10 - and 5-yr investment periods were $\$ 20$ and $-\$ 7$ (not feasible), respectively, when surplus heifer calves were sold at the $\$ 500$ base price. Using discount rates of 2.5 or $7.5 \%$ showed that the break-even price of an embryo increased to $\$ 90$ or decreased to $\$ 78$, respectively, for the 15 -yr investment period when NM $\$$ was maximized in the IVP-ET and AI systems.

\section{DISCUSSION}

This study explored the genetic, technical, and financial performance of exclusive IVP-ET systems in dairy herds compared with systems in which only AI is used. New in our study is that genetic and technical changes over time depended on 12 genetically correlated traits that are of economic importance, and phenotypic performance was modeled as a direct result of genetic changes in the traits. We assumed that the sire genetics were independent of the genetics in the herd. The results showed that genetic trends in the herd could substantially be affected by the IVP-ET system, but the default high cost of embryo transfer $(\$ 165)$ made an exclusive IVP-ET system generally less profitable than an AI system during the 15-yr investment period.

Our average annual gain of TBV of NM $\$$ of cows in the herd was $\$ 193$ for the last 11 yr of simulation, which was 0.50 SD of the assumed TBV of NM\$ (\$388). This gain is the result of the genetic trend in the sires that we assumed given. Pryce et al. (2010) predicted a genetic gain of $0.59 \mathrm{SD}$ of a composite trait for a closed population using a deterministic simulation that looked at the genetic gain achievable from IVP-ET.

\section{Alternative Modeling Choices}

Many choices had to be made when modeling the AI and IVP-ET systems that affected the results. For example, donors in the IVP-ET scenarios were selected weekly to mimic a selection procedure that can be integrated with routine weekly farm management. We assumed oocyte collection from 2 donors daily, selected from the pool of donors selected weekly.

We included a blastocyst rate of a $53 \%$, whereas blastocyst rates as high as $75 \%$ were reported by Nivet et al. (2012). The blastocyst yield was drawn stochastically from a mean of 4.25 per collection, even though an average of 5 transferable embryos from donors has 
EMBRYO TRANSFER VERSUS ARTIFICIAL INSEMINATION

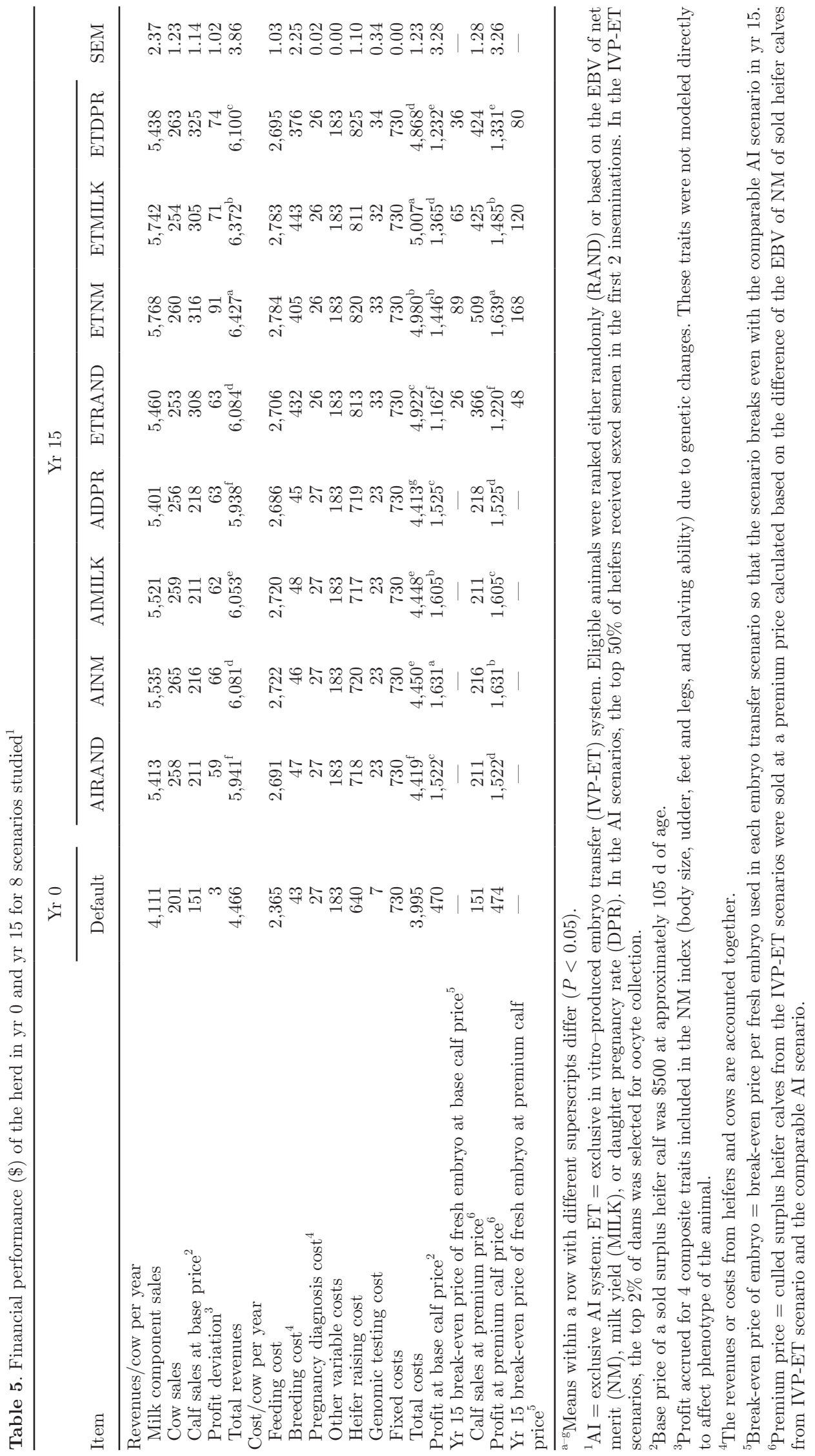


been reported (Vieira et al., 2014; Trans Ova, 2016). A greater blastocyst rate and transferable embryos yield implies that fewer donors are needed and genetic progress would be increased. On the other hand, Pontes et al. (2010) reported only 2.4 transferable embryos per collection from Holstein cows. Future work might investigate the effect of the number and quality of transferable embryos per oocyte collection on genetic, technical, and financial performance.

Another choice was putting a constraint on the number of collections per donor so superior donors could get pregnant on time. We chose not to let the genetically superior donors remain not pregnant and then be involuntarily culled due to failure to get pregnant.

When a fresh embryo was unavailable on d 8 of the estrus cycle of a potential recipient, another decision was whether she should receive a frozen embryo or wait for the next estrus to be observed. The next estrus was expected on average $13 \mathrm{~d}$ later $(21-8 \mathrm{~d})$, but estrus detection efficiency was assumed to be only $50 \%$. Because the probability of conception of fresh and frozen IVP-ET embryos was assumed the same in the model, we chose to use a frozen embryo without missing that estrus cycle.

We did not model synchronization of recipients for the sake of simplicity. Synchronization of recipients and having oocyte collection sessions once or twice per week instead of daily may be easier to implement on a farm, and its effects are a topic for future research.

Other choices of inputs that likely affected our results include, for example, maintaining a fixed annual cull rate at approximately $34 \%$, the level of estrus detection efficiency, exclusion of seasonality, discount rate, and prices. Such statistics representing the average U.S. Holstein dairy herd are not available. Therefore, our results do not necessarily represent average results for the average U.S. Holstein herd. Further sensitivity analysis might show the importance of our input choices.

Figure 4 showed that the EBV of NM $\$$ for the surplus calves from the IVP-ET system increased compared with the average EBV of NM\$ of a contemporary AI calf. Because the EBV of NM\$ of sold heifer calves were already available before the surplus heifer calves were sold, it seems reasonable that these surplus heifer calves could be sold at a price that reflects their genetic merit if such a market exists. The break-even prices of embryos further depended greatly on the assumed sale prices of surplus heifer calves. We may have undervalued bull calves produced by the IVP-ET system because in practice such calves have a greater probability of becoming future AI sires, and some could be sold at a premium price.

We assumed genomic testing of all the heifer calves born in the herd to identify the best animals to be do-

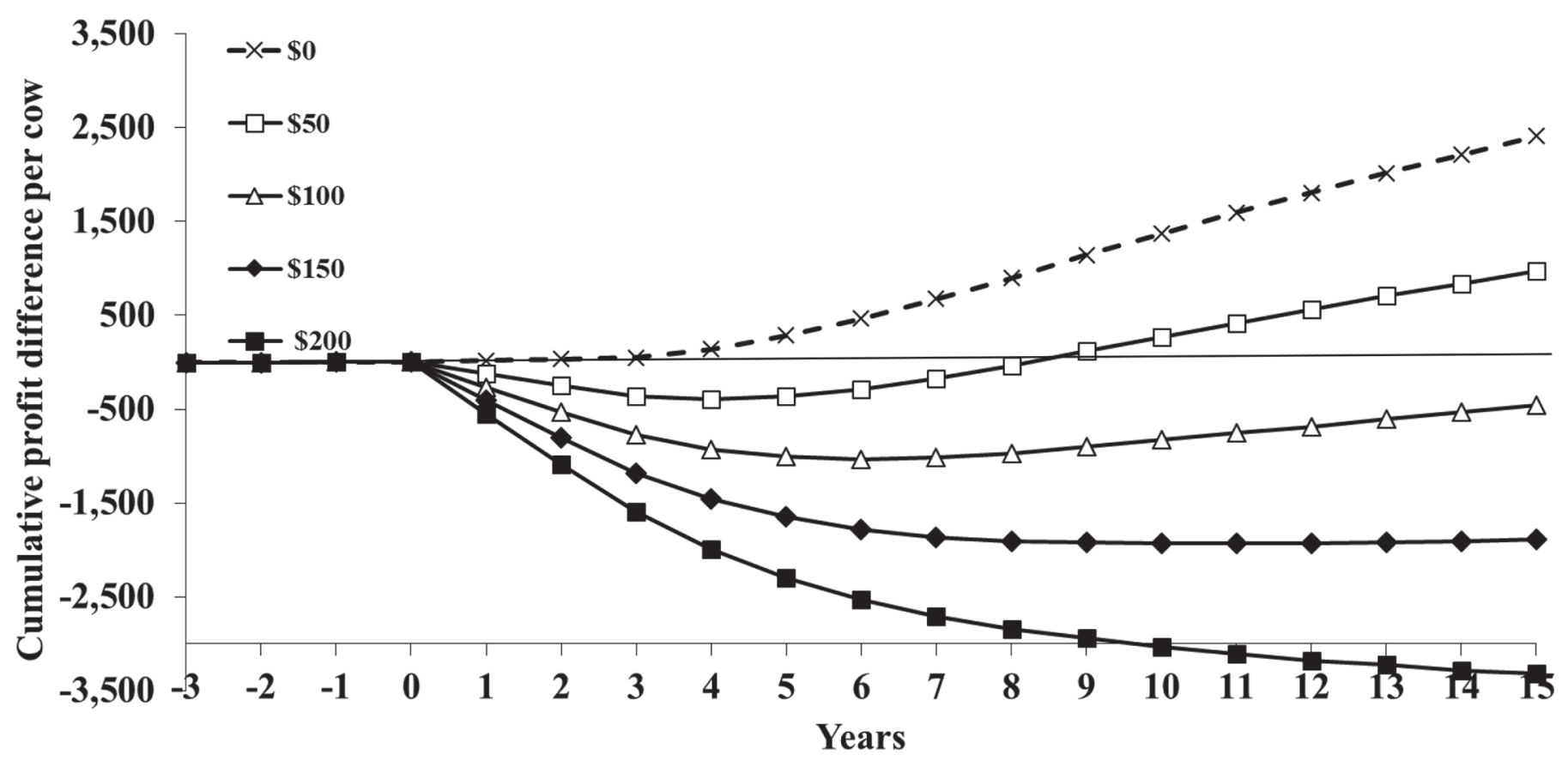

Figure 6. Net present values of the cumulative profit difference per cow of the in vitro-produced embryo transfer scenarios compared with the AI scenarios with animals ranked by EBV of net merit. Embryo transfer cost is $\$ 0, \$ 50, \$ 100, \$ 150$, or $\$ 200 /$ transfer. Premium pricing of surplus heifer calves is assumed. 
nors and the worst heifer calves to be sold. Weigel et al. (2012) showed that in order to select the best animals while limiting genomic testing costs, one should genotype the higher ranking animals based on parent average breeding values, and vice versa to select the worst animals. To improve the selection of the donors, one might genotype all heifer calves with a cheaper, lower reliability test and then genotype the higher ranking heifer calves with a higher reliability test. On the other hand, identifying surplus heifer calves to sell requires a good reliability of the lower ranking heifer calves. Therefore, genomic testing of all heifer calves seems to be a reasonable strategy.

\section{Effects of Sires and Inbreeding}

We assumed that the genetic characteristics of the sires followed the average genetic trends as predicted by VanRaden and Cole (2014). In the scenarios in which the goal was to change the TBV of milk or DPR (e.g., ETDPR), one could choose sires that are superior in these traits at the cost of a lower $\mathrm{NM} \$$. We also did not distinguish between sires used to produce embryos from sires for AI. Following VanRaden and Cole (2014), we assumed that inbreeding in the sires increased over time. Inbreeding decreased the Mendelian sampling variation in our model but not the performance of the offspring. We further did not consider individual matings to match the characteristics of the dam with those of the sires, including accounting of inbreeding (Weigel and Lin, 2000). Mc Parland et al. (2007) showed that the effect of inbreeding on phenotypic performance of inbred Holstein cows depends on the parity. Bouquet et al. (2015) showed that increasing the number of AI sires used in the population is a strategy to curb inbreeding rates without markedly reducing genetic gain.

Inbreeding is primarily a function of number of the animals selected rather than population size (Weigel, 2001). In the IVP-ET scenarios, because only approximately $45 \%$ of heifer calves born were selected as replacements compared with $70 \%$ in AI scenarios, there is a greater number of heifer calves to select from, which can reduce the amount of inbreeding expected in the herd as stated by Pryce et al. (2010).

Extensive use of IVP-ET within the herd may decrease the genetic variation over time when more females in the herd become more closely related. Granleese et al. (2015) provide a current discussion. The value of IVP-ET compared with AI would decrease if genetic variation decreases. Further, it is not clear how the phenotypic expression of genetic improvement would in practice be limited by management factors such as housing and forage quality. Such limitations would be the result of genotype by environment inter- actions. Hammami et al. (2009) offer a recent review of the evidence and magnitude for such interactions.

\section{Combining AI and IVP-ET Systems}

In this study we simulated exclusive AI or IVP-ET systems and kept the annual cow cull rate approximately constant over time. It is likely that other management decisions, such as increased culling of cows and a combination of IVP-ET with AI, might be more profitable.

The increase in productive life over time caused a gradual reduction in involuntary culling in the herd. The increase in fertility over time led to an increase in the number of potential replacement heifers. Consequently, voluntary culling in the herd increased to maintain the approximately $34 \%$ annual cow cull rate. We could have either increased or decreased our annual cow cull rate by selling fewer or more surplus heifer calves while maintaining a constant herd size. Increased genetic improvement in heifers compared with older cows would favor slightly greater cow cull rates (De Vries, 2017). The herd performance of IVP-ET systems combined with increasing voluntary cull rates is a subject for future study.

With the exclusive IVP-ET systems, approximately $55 \%$ of the heifer calves that were born were sold, and they were sold at a greater price per calf than those born in an AI system when premium pricing was in place. With smaller genetic lag and premium pricing of sold heifer calves, the profitability of the ETNM scenario was greater than that of the AINM scenario from yr 9 onward, with a lower profitability in the initial 8 yr. When return on investment during the first $15 \mathrm{yr}$ is considered, the profitability of the ETNM scenario breaks even with that of the AINM scenario only at an embryo price of $\$ 84$, which is $\$ 81$ lower than the $\$ 165$ embryo price, which we assumed to be a current market price for IVP-ET. One strategy used in practice is to produce only the heifer calves needed to replace culled cows from IVP-ET (Sjostrom, 2016). A more optimal strategy, therefore, could be to produce IVP embryos for 40 to $50 \%$ of the eligible recipients so that just the replacements are produced from IVP-ET, and all calves from AI would be sold. This would decrease the breeding costs while only slightly limiting the genetic gain achievable from IVP-ET. The optimal use of IVP-ET could also change over time - for example, fewer embryo transfers after the start of the IVP-ET system and greater use later. These more optimal strategies still need to be discovered.

The use of IVP-ET could have much greater value when selection of the sires is affected. For example, a recent study by Thomasen et al. (2016) reported that 
combining genomic testing with IVP-ET was profitable in the Danish dairy population when IVP-ET was used to produce only the bull-dams. Break-even cost per embryo transfer could be $€ 1,500$ in their case. The genetics in their model did not affect the phenotypic performance, in contrast to our model.

Our model could be further adapted to optimize the rate of genetic gain while constraining inbreeding to an acceptable level by using optimal contribution selection methods (Wray and Goddard, 1994). For that the genetic relationship between the individuals should be considered, which we have not done in the current model.

\section{CONCLUSIONS}

An IVP-ET system with donors and recipients was modeled into an existing stochastic dynamic dairy simulation that included multitrait genetics. The direction of genetic change and size of the genetic lag could be more affected by the exclusive IVP-ET system than by the exclusive AI system. The profitability of an exclusive IVP-ET system was generally lower than that of an exclusive AI system because the value of the smaller genetic lag did not compensate for the high cost of embryo transfer, especially early after the start of the IVP-ET system. Pricing of surplus heifer calves greatly affected the profitability of the IVP-ET systems. A combination of AI and IVP-ET will likely result in a positive value of some IVP-ET use in commercial dairy herds.

\section{ACKNOWLEDGMENTS}

This study was financially supported by USDA National Institute of Food and Agriculture, the Agriculture and Food Research Initiative (AFRI) grant 2013-6800420365 titled "Improving Fertility of Dairy Cattle Using Translational Genomics." We thank John Cole, USDA Agricultural Research Service (Beltsville, MD), for the clarifications he provided about the fertility traits in the Net Merit Index 2014.

\section{REFERENCES}

Bonilla, L., J. Block, A. C. Denicol, and P. J. Hansen. 2014. Consequences of transfer of an in vitro-produced embryo for the dam and resultant calf. J. Dairy Sci. 97:229-239.

Bouquet, A., A. C. Sørensen, and J. Juga. 2015. Genomic selection strategies to optimize the use of multiple ovulation and embryo transfer schemes in dairy cattle breeding programs. Livest. Sci. 174:18-25.

Chaubal, S. A., J. A. Molina, C. L. Ohlrichs, L. B. Ferre, D. C. Faber, P. E. J. Bols, J. W. Riesen, X. Tian, and X. Yang. 2006. Comparison of different transvaginal ovum pick-up protocols to optimize oocyte retrieval and embryo production over a 10 -week period in cows. Theriogenology 65:1631-1648.

De Vries, A. 2017. Economic trade-offs between genetic improvement and longevity in dairy cattle. J. Dairy Sci. 100:4184-4192.https:// doi.org/10.3168/jds.2016-11847.

De Vries, A., M. Overton, J. Fetrow, K. Leslie, S. Eicker, and G. Rogers. 2008. Exploring the impact of sexed semen on the structure of the dairy industry. J. Dairy Sci. 91:847-856.

DeJarnette, J. M., R. L. Nebel, C. E. Marshall, J. F. Moreno, C. R. McCleary, and R. W. Lenz. 2008. Effect of sex-sorted sperm dosage on conception rates in Holstein heifers and lactating cows. J. Dairy Sci. 91:1778-1785

Granleese, T., S. A. Clark, A. A. Swan, and J. H. J. van der Werf. 2015. Increased genetic gains in sheep, beef and dairy breeding programs from using female reproductive technologies combined with optimal contribution selection and genomic breeding values. Genet. Sel. Evol. 47:70.https://doi.org/10.1186/s12711-015-01513.

Haag, M., and N. Dorshorst. 2013. In vitro fertilization: A new tool for the commercial dairyman. Progressive Dairyman. Accessed May 12, 2016. http://www.progressivedairy.com/topics/a-i-breeding/ in-vitro-fertilization-a-new-tool-for-the-commercial-dairyman.

Hammami, H., B. Rekik, and N. Gengler. 2009. Genotype by environment interaction in dairy cattle. Biotechnol. Agron. Soc. Environ. $13: 155-164$.

Hansen, P. J., and J. Block. 2004. Towards an embryocentric world: The current and potential uses of embryo technologies in dairy production. Reprod. Fertil. Dev. 16:1-14.

Heikkilä, A. M., and J. Peippo. 2012. Optimal utilization of modern reproductive technologies to maximize the gross margin of milk production. Anim. Reprod. Sci. 132:129-138.

Kaniyamattam, K., M. A. Elzo, J. B. Cole, and A. De Vries. 2016 Stochastic dynamic simulation modelling including multitrait genetics to estimate genetic, technical, and financial consequences of dairy farm reproduction and selection strategies. J. Dairy Sci. 99:8187-8202. https://doi.org/10.3168/jds.2016-11136.

König, S., H. Simianer, and A. Willam. 2009. Economic evaluation of genomic breeding programs. J. Dairy Sci. 92:382-391.

Kruip, T. A. M., R. Boni, Y. A. Wurth, M. W. M. Roelofsen, and M. C. Pieterse. 1994. Potential use of ovum pick-up for embryo production and breeding in cattle. Theriogenology 42:675-684.

Mc Parland, S., J. F. Kearney, M. Rath, and D. P. Berry. 2007. Inbreeding effects on milk production, calving performance, fertility, and conformation in Irish Holstein-Friesians. J. Dairy Sci 90:4411-4419.

Meuwissen, T. H., B. J. Hayes, and M. E. Goddard. 2001. Prediction of total genetic value using genome-wide dense marker maps. Genetics 157:1819-1829.

Nivet, A. L., A. Bunel, R. Labrecque, J. Belanger, C. Vigneault, P. Blondin, and M. Sirard. 2012. FSH withdrawal improves developmental competence of oocytes in the bovine model. Reproduction 143:165-171.

Pontes, J. H. F., K. C. F. Silva, A. C. Basso, A. G. Rigo, C. R. Ferreira, G. M. G. Santos, B. V. Sanches, F. S. Faifer, F. A. M. Sterza, J. L. Schenk, and M. M. Seneda. 2010. Large-scale in vitro embryo production and pregnancy rates from Bos taurus, Bos indicus, and indicus-taurus dairy cows using sexed sperm. Theriogenology 74:1349-1355.

Pryce, J. E., M. E. Goddard, H. W. Raadsma, and B. J. Hayes. 2010. Deterministic models of breeding scheme designs that incorporate genomic selection. J. Dairy Sci. 93:5455-5466.

Qi, M., Y. Yao, H. Ma, J. Wang, X. Zhao, L. Liu, X. Tang, L. Zhang, S. Zhang, and F. Sun. 2013. Transvaginal ultrasound-guided ovum pick-up (OPU) in cattle. J. Biomim. Biomater. Tissue Eng. 18:118. https://doi.org/10.4172/1662-100X.1000118.

Ribeiro, E. S., K. N. Galvão, W. W. Thatcher, and J. E. P. Santos. 2012. Economic aspects of applying reproductive technologies to dairy herds. Anim. Reprod. 9:370-387.

Rizos, D., L. Burke, P. Duffy, M. Wade, J. F. Mee, K. J. O'Farrell, M. MacSiurtain, M. P. Boland, and P. Lonergan. 2005. Comparisons 
between nulliparous heifers and cows as oocyte donors for embryo production in vitro. Theriogenology 63:939-949.

Ruane, J. 1991. The importance of family sizes in adult multiple ovulation and embryo transfer (MOET) nucleus breeding schemes in dairy cattle. Anim. Prod. 52:33-47.

Schaeffer, L. R. 2006. Strategy for applying genome-wide selection in dairy cattle. J. Anim. Breed. Genet. 123:218-223.

Seidel, G. E. 2007. Overview of sexing sperm. Theriogenology 68:443446.

Sjostrom, L. 2016. Dairy embryos become new profit center. Dairy Herd Management. March 13, 2016. Accessed May 12, 2016. http://www.dairyherd.com/magazine/dairy-embryos-becomenew-profit-center.

Taverne, M. A. M., S. P. Breukelman, Z. Perényi, S. J. Dieleman, P. L. Vos, H. H. Jonker, L. De Ruigh, J. M. Van Wagtendonk-de Leeuw, and J. Beckers. 2002. The monitoring of bovine pregnancies derived from transfer of in vitro produced embryos. Reprod. Nutr. Dev. 42:613-624.

Thomasen, J. R., A. Willam, C. Egger-Danner, and A. C. Sørensen. 2016. Reproductive technologies combine well with genomic selection in dairy breeding programs. J. Dairy Sci. 99:1331-1340.

Trans Ova. 2016. In vitro fertilization. Accessed Sep. 7, 2016. http:// www.transova.com/our-services/ivf.php.
VanRaden, P. M., and J. B. Cole. 2014. AIP research report NM $\$ 5$ : Net merit as a measure of lifetime profit: 2014 revision. Accessed Feb. 5, 2016. http://aipl.arsusda.gov/reference/nmcalc-2014.htm.

Vieira, L. M. C. A. Rodrigues, M. F. Mendanha, M. F. Sá Filho, J. N. S. Sales, A. H. Souza, J. E. P. Santos, and P. S. Baruselli. 2014. Donor category and seasonal climate associated with embryo production and survival in multiple ovulation and embryo transfer programs in Holstein cattle. Theriogenology 82:204-212.

Weigel, K. A. 2001. Controlling inbreeding in modern breeding programs. J. Dairy Sci. 84:E177-E184

Weigel, K. A., P. C. Hoffman, W. Herring, and T. J. Lawlor Jr.. 2012. Potential gains in lifetime net merit from genomic testing of cows, heifers, and calves on commercial dairy farms. J. Dairy Sci. 95:2215-2225.

Weigel, K. A., and S. W. Lin. 2000. Use of computerized mate selection programs to control inbreeding of Holstein and Jersey cattle in the next generation. J. Dairy Sci. 83:822-828.

Wilson, R. D., P. M. Fricke, M. L. Leibfried-Rutledge, J. J. Rutledge, C. M. S. Penfield, and K. A. Weigel. 2006. In vitro production of bovine embryos using sex-sorted sperm. Theriogenology 65:10071015.

Wray, N. R., and M. E. Goddard. 1994. Increasing long-term response to selection. Genet. Sel. Evol. 26:431-451. 